\title{
Simple Rail-to-Rail Constant-Transconductance Input Stage Operating in Strong Inversion
}

\author{
Vladimir I. Prodanov \& Michael M. Green \\ Department of Electrical Engineering \\ State University of New York \\ Stony Brook, NY 11794-2350
}

\begin{abstract}
A CMOS op-amp input and gain stage suitable for low voltage operation are introduced. The input stage operates in strong inversion and has common-mode range beyond rail-to-rail. It uses two complementary differential pairs connected in parallel. The common-mode dependent current biasing employs only four transistors, does not require additional voltage references, current switches and/or current mirrors and does not increase the minimum required supply voltage. The variation of the net transconductance is approximately $15 \%$ over the entire common-mode range. The gain-stage has constant output resistance. In addition it reduces the variation of the net transconductance due to variations in $\mu_{n} / \mu_{p}$ ratio.
\end{abstract}

\section{INTRODUCTION}

A widely used technique for obtaining a rail-to-rail input range, when designing low-voltage op-amps, is to connect two complementary differential pairs in parallel as shown in Fig. 1(a). In this way one guarantees that for any commonmode input voltage at least one of the differential pairs will operate properly. This simple topology is rarely used, however, because its net transconductance $g_{m_{T}}$ varies by a factor of two over the common-mode input range. In midsupply range, where both pairs operate, the net transconductance is given by:

$$
g_{m_{T}}=g_{m_{n}}+g_{m_{p}}
$$

However, when the input common-mode voltage approaches the positive (negative) rail $g_{m_{T}}$ reduces to $g_{m_{n}}\left(g_{m_{p}}\right)$ respectively. This variation does not allow optimal frequency compensation of multi-stage op-amps and also increases their distortion.

Since the individual differential-pair transconductances $g_{m_{n}}$ and $g_{m_{p}}$ are well-defined functions of the of the tail currents $I_{n}$ and $I_{p}$, respectively, a general method for obtaining common-mode-independent net transconductance is to employ common-mode - dependent current biasing. In other words, we balance the reduction in $g_{m_{n}}\left(g_{m_{p}}\right)$ (caused by the reduction of $I_{n}\left(I_{p}\right)$ when $V_{i n c M}$ approaches $\left.V_{s s}\left(V_{d d}\right)\right)$ by increasing $I_{p}\left(I_{n}\right)$ (e.g. Fig. 1(b)).

In the case of an input stage using BJT's or MOS transistors in weak inversion the requirement that $g_{m_{T}}$ be independent of $V_{\text {in } C M}$ translates into the following, simple to implement, current-biasing requirement:

$$
I_{n}+I_{p}=\text { const. }
$$

If MOS transistors in strong inversion are to be used in such a rail-to-rail input stage the current biasing requirement is:

$$
\sqrt{I_{n}}+\sqrt{I_{p}}=\text { const. for } \beta_{n}=\beta_{p}
$$

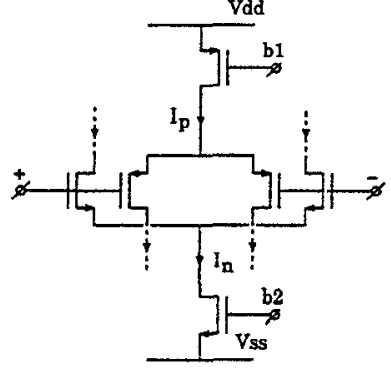

a)

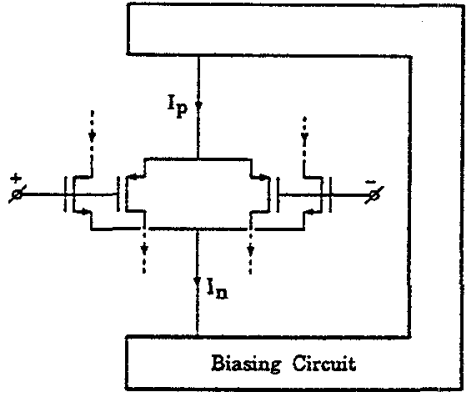

b)
Figure 1: (a) Simple rail-to-rail input stage; (b) Conceptual. schematic of constant- $g_{m}$ rail-to-rail input stage.

and, more general:

$$
\sqrt{\left(2 / \beta_{n}\right) I_{n}}+\sqrt{\left(2 / \beta_{p}\right) I_{p}}=\text { const. for } \beta_{n} \neq \beta_{p}
$$

The main differences between various rail-to-rail input stage topologies reported in the literature is the employed biasing scheme. Those schemes targeting the MOS-strong inversion case can lorsely be classified as either exact or approximate. All schemes realizing equations (3) or (4) [1], [2], [3] are considered exact, because under ideal conditions (perfect matching and no second order effects) they result in a constant net transconductance. The approximate schemes are baised on the assumption that the tail currents $I_{n}, I_{p}$ take either their nominal value or are completely turned off. Those schemes are usually implemented by use of a simple $1: 3$ current mirrors [3], [4] and can reduce the $g_{m_{T}}$-variation to within $15 \%$. Unfortunately, most of the reported strong inversion biasing circuits have high complexity.

In Section II we present a simple approximate scheme which requires only four additional transistors and has a $g_{m_{T}}$ variation of approximately $15 \%$. In Section III a gain stage which has constiant output resistance is introduced. Also, it reduces the variations of the effective net transconductance caused by variations in the mobility ratio $\mu_{n} / \mu_{p}$.

\section{The Proposed Rall-To-RaIl InPut Stage}

The input stage, shown in Fig. 2(a), is topologically identical to that reported in [5]. However, the aspect ratios of the four additional transistors in the Fig. 2(a) circuit is three times that of the corresponding differential-pair transistors. The nominal value of the tail currents $I_{S_{n}}$ and 
$I_{S_{p}}$ is $4 I_{0}$ and must be selected sufficiently large to ensure strong-inversion operation. The aspect ratios of the $n$ and $p$-channel transitory are selected such that $\beta_{n}=\beta_{p}=\beta$. Under this conditions the Fig. 2(a) circuit guarantees railto-rail operation with a $15 \%$ variation in $g_{m_{T}}$. To show this let us first consider the operation of the $M_{5}-M_{8}$ quartet. As discussed in [5] if a CMOS current source $\left(M_{5}-M_{6}\right)$ is connected in series with a current $\operatorname{sink}\left(M_{7}-M_{8}\right)$, the pair which must carry the higher current is forced into the triode region and thus the smaller of the two currents is conducted. For our case:

$$
I_{x}=\min \left(\frac{3}{4} I_{S_{n}}, \frac{3}{4} I_{S_{p}}\right)=\frac{3}{4} \min \left(I_{S_{n}}, I_{S_{p}}\right)
$$

Hence, the current $I_{x}$ and the currents $I_{p}=I_{S,}-I_{x}$ $\left(I_{n}=I_{S_{n}}-I_{x}\right)$ conducted by the differential-pair transistors $M_{1,2}\left(M_{3,4}\right)$ of the Fig. 2(a) stage are,

$$
\text { 1) } V_{i n_{C M}} \text { close to } V_{s s}: \quad \begin{aligned}
& I_{x}=3 / 4 I_{S_{n}}=0 \\
& I_{n}=1 / 4 I_{S_{n}}=0 \\
& I_{p}=I_{S_{p}}-I_{x}=4 I_{0}-I_{x}=4 I_{0} \\
& \\
& I_{x}=3 I_{0}
\end{aligned}
$$

2) $V_{\text {in } C M}$ near mid-supply: $I_{n}=I_{S_{n}}-I_{x}=4 I_{0}-3 I_{0}=I_{0}$ $I_{p}=I_{S_{p}}-I_{x}=4 I_{0}-3 I_{0}=I_{0}$

3) $V_{\text {incM }}$ close to $V_{d d}$ :

$$
I_{z}=3 / 4 I_{S_{p}}=0
$$

$$
\begin{aligned}
& I_{n}=I_{S_{n}}-I_{x}=4 I_{0} \\
& I_{p}=1 / 4 I_{S_{p}}=0
\end{aligned}
$$

Thus for the above three regions of operation, where the tail currents $I_{S_{n}}, I_{S_{p}}$ have their nominal $\left(4 I_{0}\right)$ value or have zero value, the total transconductance (see equation (1)) is the same and given by:

$$
g_{m_{T}}=2 \sqrt{(2 / \beta) I_{0}} \text { when } \beta_{n}=\beta_{p}=\beta
$$

For the transition regions, where $0<I_{S_{n, p}}<4 I_{0}, g_{m_{T}}$ is not constant and slightly higher than the above value. The maximum $g_{m_{T}}$ deviation can be calculated and is approximately $15 \%$.

The Fig. 2(a) rail-to-rail input stage was simulated using HSPICE and BSIM2 (level 13) models for MOSIS 2-micron ORBIT Analog Process. The size of the transistors and the value of the constant bias current $4 I_{0}$ were as indicated on the schematic. Fig. 2(b) shows the variation of $I_{n}$ and $I_{p}$ bias currents as the $V_{i n_{C M}}$ is swept from $V_{S S}$ to $V_{d d}$. The three regions (near- $V_{S S}$, midusupply and near- $V_{d d}$ ) where $I_{n}$ and $I_{p}$ must remain constant are evident. The non-zero slope of those regions is due to the finite output resistance of the used transistors. In Fig. 2(c) the simulated individual $\left(g_{m_{n}}, g_{m_{p}}\right)$ and net transconductance $\left(g_{m_{T}}\right)$ are plotted v.s. the input common-mode voltage. As expected, there are two "bumps" in the $g_{m_{T}}$ plot corresponding to the two transition regions. The small "glitch" present within each "bump" can not be predicted if the simple square-law relation is used to model the voltage-current behavior of the MOS transistor. Since "glitches" occur when one of the bias currents $\left(I_{n}\right.$ or $\left.I_{p}\right)$ has relatively low value, they are

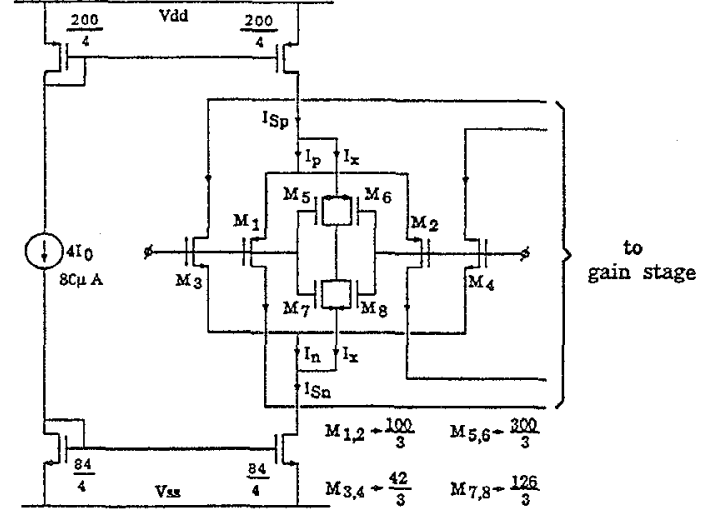

(a)

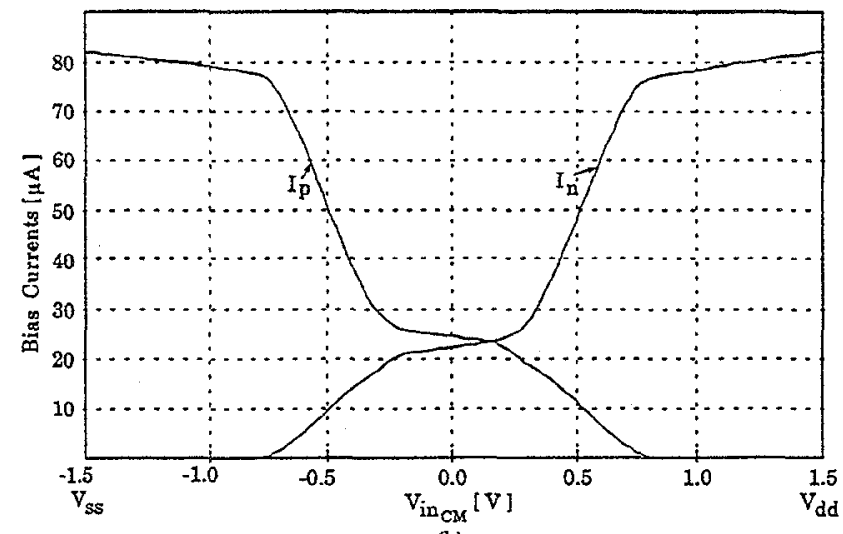

(b)

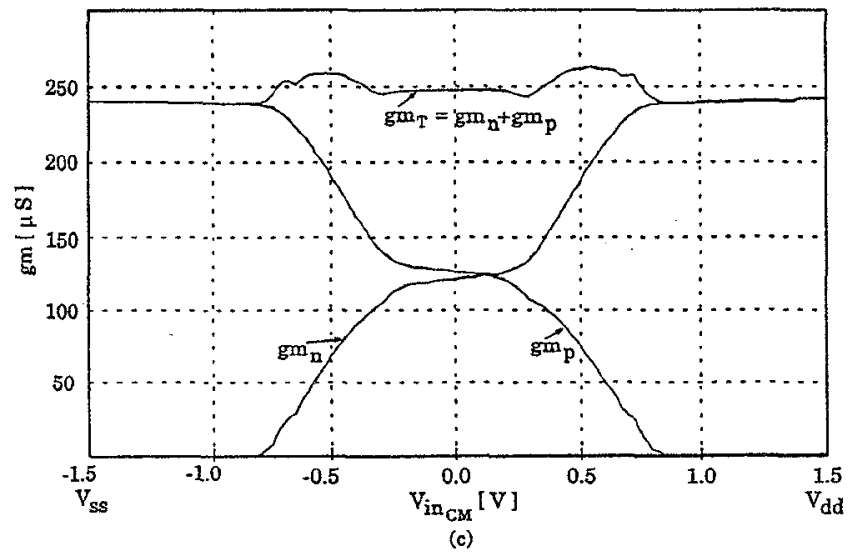

Figure 2: Proposed Rail-to-Rail Input Stage: (a) Schematic; (b) Bias Currents v.s. $V_{\text {in } C M}$; (c) Individual and Net Transconductance v.s. $V_{\text {in } C M}$;

most likely due to one of the differential pairs entering moderate and then weak inversion region of operation.

Because of finite $r_{0}$ effects, $I_{n}$ and $I_{p}$ are slightly larger than $20 \mu \mathrm{A}$ in the mid-supply region. For this reason the net transconductance in this region is slightly higher than that in the near-rail regions. As is the case with many other rail-to-rail input stages, the one presented in this 


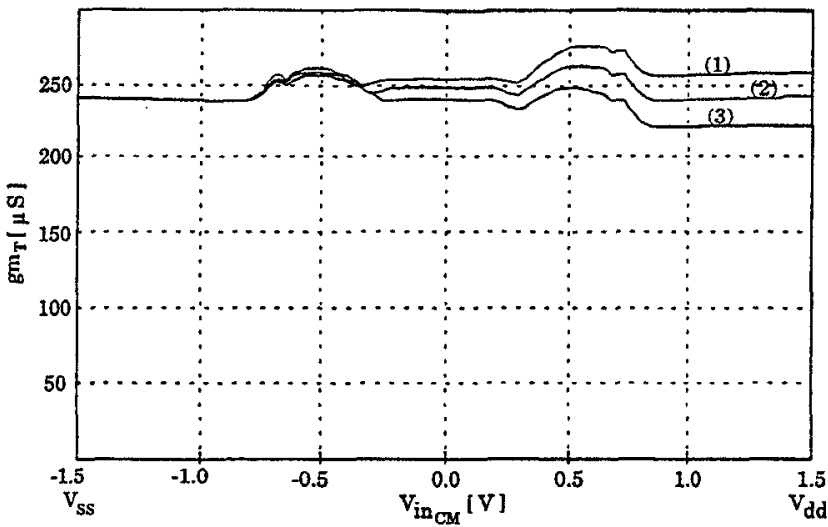

Figure 3: Net transconductance v.s. $V_{\text {incm }}$ for three different values of $\frac{\mu_{n}}{\mu_{p}}:$ (1) "actual" $\frac{\mu_{n}}{\mu_{p}}$ is $15 \%$ higher than the one used in the design; (2) "actual" $\frac{\mu_{n}}{\mu_{p}}=\operatorname{used} \frac{\mu_{n}}{\mu_{p}} ;(3)$ "actual" $\frac{\mu_{n}}{\mu_{p}}$ is $15 \%$ less than the used one;

paper relies on matching the transconductance parameter $(\beta)$ of the used $n$-channel transistors to that of the used p-channel transistors. If the ratio between the mobility of the n-channel transistors and the mobility of the p-channel transistors $\mu_{n} / \mu_{p}$ is exactly known then $\beta_{n}$ can be made very close to $\beta_{p}$ by simply sizing the transistors whose $\beta$ 's are to be matched in accordance with: $W_{n}=W_{p} \frac{\mu_{p}}{\mu_{n}}$ for $L_{n}=L_{p}$. Unfortunately, for a given process from one run to another the ratio of the mobilities could vary as much as $30 \%$ from its nominal value [1] used to determine "the best" n-channel and p-channel aspect ratios. To illustrate the effect this variation would have on the net transconductance of the proposed input stage two additional simulations were performed. The width of the n-channel devices was changed to $48 \mu$ and $36 \mu$ - that is, a change of $+15 \%$ and $-15 \%$ from its nominal $42 \mu$ width. Since $\beta$ equality is achieved for $\frac{W_{n}}{W_{p}}=\frac{42}{100}$, transistor having $W_{n}$ equal to $48 \mu(36 \mu)$ would be equivalent to transistor having $W=42$ (the nominal value) and $\mu$ increased (decreased) by $15 \%$. Fig. 3 shows the results from the simulations.

\section{The Gain Stage}

Fig. 4 shows single-stage unbuffered op-amp which uses the input stage described in the previous section. The gain stage consists of two MOS-R current mirrors and a floating current source. Here, as in many other reported in the literature rail-to-rail topologies, in addition to providing voltage gain the gain stage is used to sum the small signal (differential) currents generated by the two input-stage differential pairs. However, there are some unique properties possessed by this gain stage. First, its output resistance and thus the op-amp's gain - is independent of the level of the injected by the input stage common-mode currents $\left(I_{n}\right.$, $I_{p}$ ). As a result the distortion caused by common-mode dependent gain is kept at its minimum. To show that the output resistance is constant it is sufficient to show that the output resistance of each current mirror is constant.

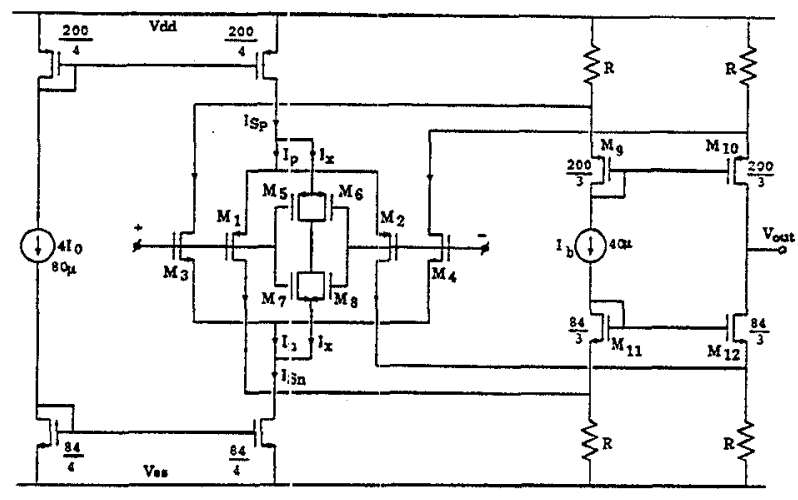

Figure 4: Single-stage unbuffered op-amp.

The output resistance of the Fig. 5 current mirror, given by:

$$
r_{\text {out } t_{2}}=\left(1+g_{m_{12}} R\right) r_{o_{12}}
$$

would be constant if both the transconductance and the output resistance $\left(g_{m_{12}}\right.$ and $\left.r_{o_{12}}\right)$ of $M_{12}$ transistor are constant. The latter is guaranteed when the current conducted by $M_{12}\left(I_{M_{12}}\right)$ is constant. The KVL equation written for the Fig. 5 MOS-R current mirror:

$$
R\left(\frac{1}{2} I_{p}+I_{b}\right)+\sqrt{\frac{2}{\beta} I_{b}}=R\left(\frac{1}{2} I_{p}+I_{M_{12}}\right)+\sqrt{\frac{2}{\beta} I_{M_{22}}}
$$

where $\beta=\beta_{11}=\beta_{12}$, shows that indeed:

$$
I_{M_{12}}=I_{b}=\text { const. }
$$

Second, due to the finite $g_{m_{11,12}}$ and $R$ only a portion of the differential input current will be transferred to the output (see the equivialent circuit shown in Fig. 5(b)):

$$
i_{\text {out }}=\frac{g_{m_{11,12}} R}{1+g_{m_{11,12}} R} i_{\text {in }}
$$

This property seems undesirable because it reduces the effective transconductaace $g_{m_{T},}^{\prime}$ of this single-stage op-amp (e.g. Fig. 6). More importantly, if the factors $\frac{g_{m 9,10} R}{1+g_{m_{9,10}} R}$ and $\frac{g_{m_{11,12}} R}{1+g_{m_{11,12}} R}$ are not equal, $g_{m_{T}}^{t}$ will not be constant
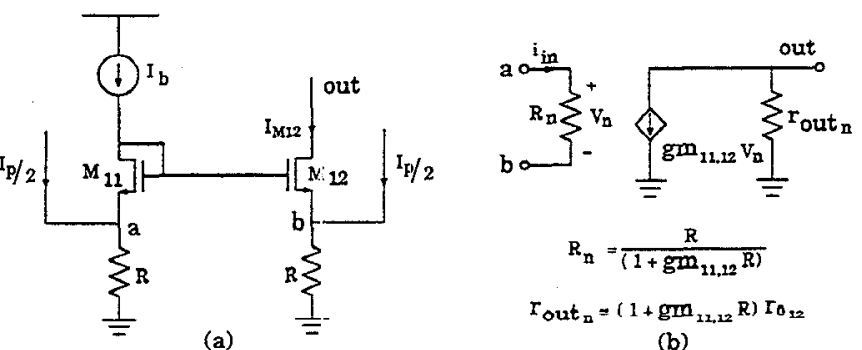

Figure 5: (a) n-channel R-MOS current mirror; (b) small differential signal equivalent of (a). 

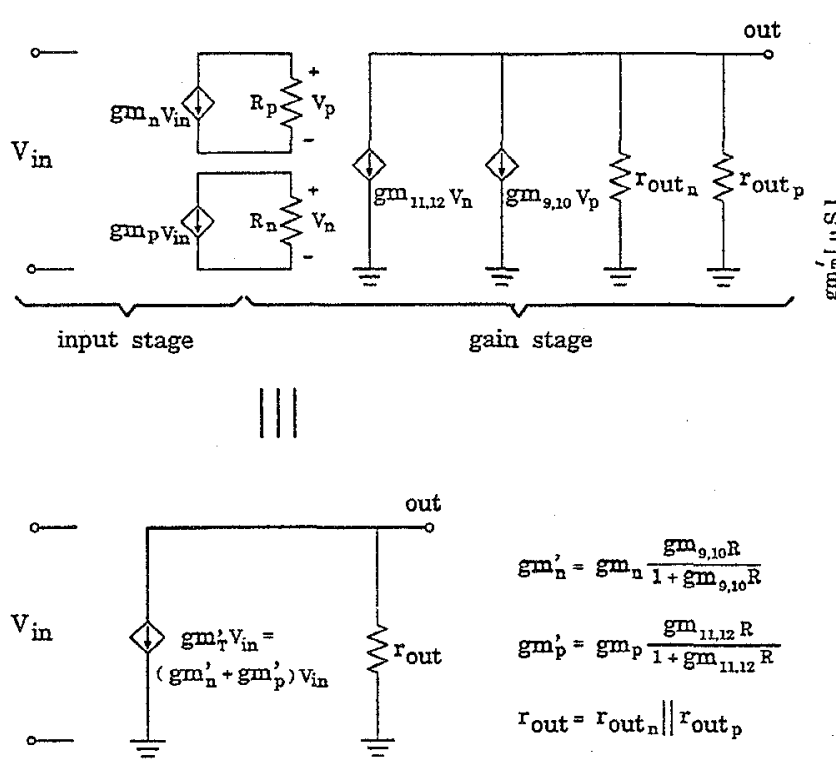

Figure 6: Simplified equivalent circuits of Fig. 4 singlestage op-amp.

over the common-mode input range. Achieving equality of the above current gain expressions can be accomplished be making $g_{m_{0,10}}=g_{m_{11,12}}=g_{m}$. This is done as in the input stage by sizing $M_{9}, M_{10}$ and $M_{11}, M_{12}$ so their $\beta$ 's are identical.

The advantage of this gain stage over others becomes obvious only when the variations in $g_{m_{T}}$ and $g_{m_{T}}^{\prime}$ due to differences between the actual ratio of the mobilities and the one used to carry out the design are compared. The smaller the product $g_{m} R$ the lower the $g_{m_{T}}^{\prime}$-variation. However, this product should not be made lower than unity in order to retain a sufficiently large overall op-amp gain. As can be seen from the plots shown in Fig. 7, the change of $\mu_{n}$ effects the transconductance of both the near- $V_{s s}$ and near- $V_{d d}$ range in same direction which results in lower relative variation within each curve. This can be explained with the fact that now each individual $g_{m_{n}}^{\prime}$ and $g_{m_{p}}^{\prime}$ is determined by the transconductances of both n-channel and p-channel transistors.

If higher output resistance is desired the simple $M_{9}, M_{10}$ and $M_{11}, M_{12}$ current mirrors can be replaced by highswing cascoded current mirrors without altering the properties of the gain stage.

\section{Conclusions}

A simple rail-to-rail input stage operating in strong inversion was presented. Its net transconductance variation is approximately $15 \%$ over the entire common-mode range. A new gain stage was introduced. It was shown that the transconductance variation in this gain stage caused by imperfect $\beta$-matching is reduced as compared to previous techniques.

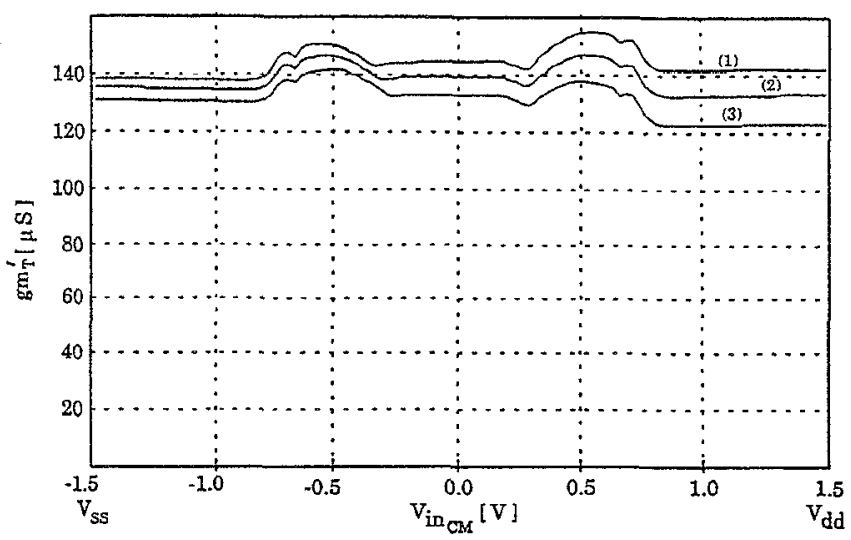

Figure 7: Effective net transconductance v.s. $V_{i n_{C M}}$ for three different values of $\frac{\mu_{n}}{\mu_{p}}$ : (1) "actual" $\frac{\mu_{n}}{\mu_{p}}$ is $15 \%$ higher than the one used in the design; (2) "actual" $\frac{\mu_{n}}{\mu_{p}}=$ used $\frac{\mu_{n}}{\mu_{p}}$; (3) "actual" $\frac{\mu_{n}}{\mu_{p}}$ is $15 \%$ less than the used one;

\section{REFERENCES}

[1] S. Sakurai and M. Ismail, LOW-VOLTAGE CMOS OPERATIONAL AMPLIFIERS: Theory, Design and Implementation. Kluwer Academic Publishers, 1995.

[2] K. Nagaraj, "Constant transconductance CMOS amplifier input stage with rail-to-rail input common mode voltage range," IEEE Transactions on Circuits and Systems - Part II, vol. 42, pp. 366-368, 1995.

[3] R. Hogervorst et al., "CMOS low-voltage operational amplifiers with constant- $g_{m}$ rail-to-rail input stage," Proc. of ISCAS, vol. 6, pp. 2876-2879, 1992.

[4] A. L. Coban and P. E. Allen, "A low-voltage CMOS op amp with rail-to-rail constant- $g_{m}$ input stage and high-gain output stage," Proc. of ISCAS, vol. 2, pp. 1548-1551, 1995.

[5] J. H. Botma, R. F. Wassenaar and R. J. Wiegerink, "Simple rail-to-rail low-voltage constant-transconductance CMOS input stage in weak inversion," Electronics Letters, vol. 29, no. 12, pp. 1145-1147, June 1993. 